\title{
Effectiveness of Functional Endoscopic Sinus Surgery in Improving Quality of Life - A Comparative Study Among Smokers and Non Smokers with Chronic Rhinosinusitis
}

\section{Ashok Vivekanand* and Lyra Joy}

Senior Resident, Department of Otorhinolaryngology and Head and Neck Surgery, Karnataka Institute of Medical Sciences, Hubballi, Karnataka, India

*Corresponding Author: Ashok Vivekanand, Senior Resident, Department of Otorhinolaryngology and Head and Neck Surgery, Karnataka Institute of Medical Sciences, Hubballi, Karnataka, India.
Received: September 13, 2021

Published: November 30, 2021

(C) All rights are reserved by Ashok

Vivekanand and Lyra Joy.

\begin{abstract}
Objectives: The objectives of this study was to evaluate the effectiveness of functional endoscopic sinus surgery in improving quality of life using Sino nasal outcome test 22 and to compare how effective was endoscopic sinus surgery among smokers and non smokers.

Methods: This was a prospective study, conducted at a tertiary care centre were 100 patients with chronic rhinosinusitis who were planned to undergo endoscopic sinus surgery were included in the study. Patients were asked to complete pre operatively the Sino nasal outcome test 22 questionnaire and a questionnaire to know their smoking habits. The Sino nasal outcome test 22 questionnaire details were taken at end 6th month of post operative period and data were analysed.

Results: The results were that, there was significant reduction in Sino nasal outcome test 22 scores post operatively; however there was no significant difference in the reduction pattern among smokers and non smokers.

Conclusion: Endoscopic sinus surgery is an effective treatment in improving quality of life in patients with chronic rhinosinusitis and Sino nasal outcome test 22 can be considered as a tool to evaluate the quality of life and effectiveness of functional endoscopic sinus surgery. The role of smoking in development of chronic rhinosinusitis is to be evaluated further.

Keywords: Chronic Rhinosinusitis; Cigarette Smoke; Quality of Life; Functional Endoscopic Sinus Surgery; Sino Nasal Outcome Test 22
\end{abstract}

\section{Abbreviations}

CRS: Chronic Rhinosinusitis; FESS: Functional Endoscopic Sinus Surgery; QoL: Quality of Life; SNOT 22: Sinonasal Outcome Test 22

\section{Introduction}

Rhino sinusitis is defined as the inflammation of the lining of the nose and paranasal sinuses characterised by one or more of the following symptoms: nasal congestion, rhinorrhea, sneezing, itching, hyposmia/anosmia, facial pain and headache. Chronic rhinosinusitis (CRS) is described when the duration of symptoms is more than 12 weeks, persistent inflammatory changes on imaging
4 weeks after starting appropriate medical therapy with no intervening acute episode [1].

Failure of acute infection to resolve is the most important cause for going into chronicity. The normal ciliary epithelium will be destroyed following acute infection leading to pooling and stagnation of secretions in paranasal sinuses, thus providing a favourable condition for microbial growth [2].

Several factors like dust, smoke, cigarette smoke, persistence of nasal infection because of tonsillitis, adenoid hypertrophy, deviated nasal septum, synechiae etc. predispose to CRS. 
These factors invariably disrupt the normal mucociliary clearance (MCC) mechanism which helps in clearing the sinonasal secretions and also one of the primary defence mechanisms in protecting the lower airway. This explains how these environmental toxins predispose to sinusitis and other lower respiratory diseases [3].

The most well studied environmental toxin in relation to CRS is cigarette smoke [4]. Smoking causes increased oxidative stress leading to airway inflammation, defective anion transport, abnormalities in cilia ultrastructure, reduction in ciliary beat frequency and ciliary genesis and evidence of nasal inflammation with increased number of inflammatory cells and pro inflammatory cytokines like Interleukins 6 and 8 [5].

Adult CRS is one of the most common healthcare problems for which individual approaches healthcare centres leading to high direct medical costs [6]. Along with symptoms, CRS causes significant functional and emotional impairment which ultimately impacts the Quality of life (QoL). One's health status may not reflect the QoL in most of cases. Several tools have been developed across the world to assess the QoL. These help in better evaluation of morbidity, disease progression and impact of different treatment methods. Adequate validation of such tools is essential in allowing comparisons among different populations. A widely used QoL questionnaire is Sino Nasal Outcome Test-22 (SNOT-22). It has been found that patients with CRS have more impairment in social function as compared to conditions like angina, chronic obstructive pulmonary diseases, congestive heart failure and back pain [7].

The functional endoscopic sinus surgery popularized by Messerklinger and Stammberger is considered as a surgical option in patients with CRS not responding to maximal medical therapy which is up to 3 months after diagnosing CRS. The treatment regime should include topical/systemic steroids, antibiotics and decongestants [8].

This study aims to assess the effectiveness of FESS in improving QoL in CRS patients using SNOT-22 and also to compare the QoL among smokers and non smokers with CRS.

\section{Materials and Methods}

It is a prospective study where 100 patients with chronic rhinosinusitis were included during the study period November 2017 to
May 2019 at a tertiary care centre. CRS patients of age more than 18 years of either sex willing to give informed consent and willing for follow up for a period of six months post operatively were included in the study. Patients with systemic illness and those undergone surgical procedure for CRS or other nasal conditions previously were excluded from the study.

After receiving the institute ethical committee approval, patients in whom CRS disease persisted after maximum conservative medical therapy and undergone FESS as a treatment option were included in the study.

Patients were asked to fill two questionnaires- a subjective SNOT 22 questionnaire and questionnaire to know the smoking habits, pre operatively and six months post operatively. Those who had the habit of smoking were advised to quit smoking, as smoking can be a contributing factor for their condition.

\section{Results}

The study population included 61 males and 39 females (n = 100). Among 100 patients,29 were smokers, out of which 27 males(93.10)\% and 2 females (06.89\%). The non smoking population included 71 patients 34 males (47.88\%) and 37 females $(52.11 \%)$ (Figure 1,2). When each symptom in SNOT 22 was compared pre and post operatively in both groups, FESS was found to be useful in alleviating each symptom score in both the groups (Table 1,2). The difference in mean values (pre operative mean value- post operative mean value) of each symptom was compared in both groups. This comparison showed smokers had better improvement with the surgical procedure than non smokers in the major symptoms associated with CRS, such as need to blow nose, nasal blockage and runny nose. The study also found that the sleep associated symptoms showed more reduction in mean value following surgery among smokers as compared to non smokers. This may be due to the fact that smokers had more troublesome sleep pre operatively. The mean average SNOT 22 score comparison showed that FESS was equally effective in both the smoking and non smoking patients groups, which was supported after doing paired $t$ test, where $t$ value $(-23.11)$ was not in the $95 \%$ critical value accepted range $(-6.318-\infty)$ and hence showed no significance in comparing both the groups (Table 1). The overall SNOT 22 was compared among those who had tried in either quitting or reducing smoking habit and continued to smoke as in pre operative period. The pre operative score was higher in those who had not tried quit-

Citation: Ashok Vivekanand and Lyra Joy. "Effectiveness of Functional Endoscopic Sinus Surgery in Improving Quality of Life - A Comparative Study Among Smokers and Non Smokers with Chronic Rhinosinusitis". Acta Scientific Otolaryngology 3.12 (2021): 56-61. 
Effectiveness of Functional Endoscopic Sinus Surgery in Improving Quality of Life - A Comparative Study Among Smokers and Non Smokers with Chronic Rhinosinusitis

ting smoking at any point of time in life, however the postoperative improvement was comparably equal in both groups and showed no statistical significance in effectiveness of FESS while comparably both the groups, suggesting that FESS was equally effective in both groups.

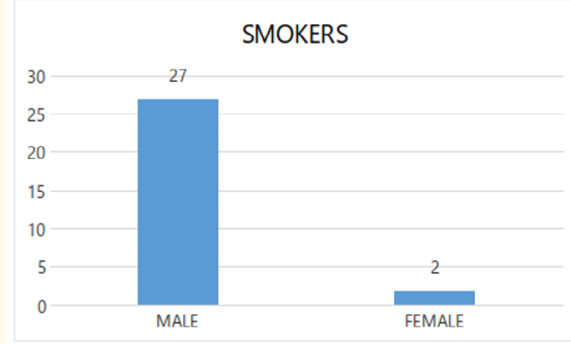

Figure 1: Gender distribution in smoking group.

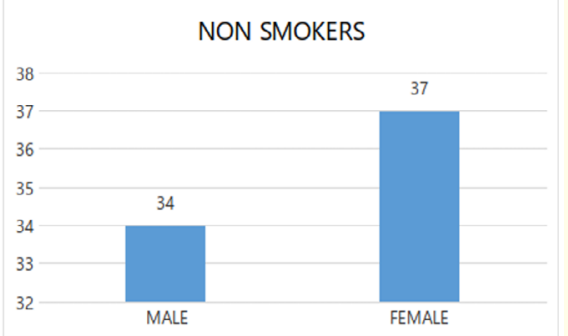

Figure 2: Gender distribution in non smoking group.

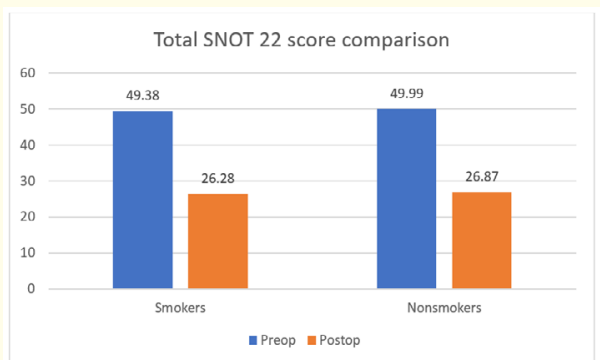

Figure 3: Overall SNOT 22 score comparison among both groups.

\begin{tabular}{|l|c|c|c|}
\hline \multirow{2}{*}{ SNOT 22 QUESTION } & \multicolumn{3}{|c|}{58} \\
\cline { 2 - 4 } & $\begin{array}{c}\text { Pre operative } \\
\text { mean value }\end{array}$ & $\begin{array}{c}\text { Post operative } \\
\text { mean value }\end{array}$ & Z value \\
\hline Need to blow nose & 2.69 & 1.28 & 4.768 \\
\hline Nasal blockage & 3.24 & 1.72 & 4.354 \\
\hline Sneezing & 2.79 & 1.41 & 4.479 \\
\hline Runny nose & 2.59 & 1.17 & 4.77 \\
\hline Cough & 2.07 & 0.97 & 4.463 \\
\hline Post nasal discharge & 1.93 & 0.83 & 4.235 \\
\hline Thick nasal discharge & 2.90 & 1.55 & 4.284 \\
\hline Ear fullness & 2.45 & 1.10 & 4.273 \\
\hline Dizziness & 2.07 & 1.31 & 3.055 \\
\hline Ear pain & 2.07 & 1.28 & 3.734 \\
\hline Facial pain & 2.66 & 1.52 & 3.841 \\
\hline Decreased taste/smell & 2.93 & 1.66 & 4.189 \\
\hline Difficulty falling asleep & 2.14 & 1.17 & 3.458 \\
\hline Wake up night & 2.03 & 1.48 & 2.173 \\
\hline Lack of a good night's & 1.83 & 0.76 & 4.382 \\
\hline sleep & 1.83 & 0.76 & 4.212 \\
\hline Wake up tired & 1.97 & 1.31 & 2.742 \\
\hline Fatigue & 1.97 & 0.83 & 4.332 \\
\hline Reduced productivity & 2.03 & 1.07 & 3.557 \\
\hline Reduced concentration & 1.62 & 1.10 & 2.065 \\
\hline Frustrated/irritable & 1.90 & 1.14 & 3.085 \\
\hline Sad & 1.69 & 0.86 & 3.452 \\
\hline Embarrassed & & & \\
\hline
\end{tabular}

Table 1: Symptom score comparison in smoking group.

\begin{tabular}{|l|c|c|c|}
\hline SNOT 22 QUESTION & $\begin{array}{c}\text { Pre operative } \\
\text { mean value }\end{array}$ & $\begin{array}{c}\text { Post operative } \\
\text { mean value }\end{array}$ & Z value \\
\hline Need to blow nose & 2.75 & 1.42 & 7.175 \\
\hline Nasal blockage & 3.03 & 1.61 & 7.193 \\
\hline Sneezing & 2.82 & 1.48 & 7.167 \\
\hline Runny nose & 2.72 & 1.32 & 7.331 \\
\hline Cough & 1.77 & 0.83 & 7.606 \\
\hline Post nasal discharge & 2.13 & 0.89 & 6.855 \\
\hline Thick nasal discharge & 2.85 & 1.38 & 6.123 \\
\hline Ear fullness & 2.49 & 1.08 & 4.605 \\
\hline Dizziness & 2.10 & 1.20 & 5.369 \\
\hline Ear pain & 2.45 & 1.24 & 5.676 \\
\hline
\end{tabular}


Effectiveness of Functional Endoscopic Sinus Surgery in Improving Quality of Life - A Comparative Study Among Smokers and Non Smokers with Chronic Rhinosinusitis

\begin{tabular}{|l|c|c|c|}
\hline Facial pain & 2.68 & 1.38 & 6.229 \\
\hline Decreased taste/smell & 2.62 & 1.62 & 4.989 \\
\hline Difficulty falling asleep & 2.04 & 1.23 & 5.349 \\
\hline Wake up night & 1.93 & 1.23 & 3.858 \\
\hline $\begin{array}{l}\text { Lack of a good night's } \\
\text { sleep }\end{array}$ & 1.97 & 1.20 & 4.334 \\
\hline Wake up tired & 1.90 & 0.89 & 6.292 \\
\hline Fatigue & 2.03 & 1.30 & 4.138 \\
\hline Reduced productivity & 1.96 & 0.86 & 6.293 \\
\hline Reduced concentration & 2.15 & 1.37 & 4.582 \\
\hline Frustrated/irritable & 1.99 & 1.13 & 5.238 \\
\hline Sad & 1.90 & 0.97 & 6.484 \\
\hline Embarrassed & 1.65 & 0.86 & 5.222 \\
\hline
\end{tabular}

Table 2: Symptom score comparison in non smoking group.

\begin{tabular}{|l|c|c|}
\hline SNOT 22 QUESTION & $\begin{array}{c}\text { Difference in } \\
\text { mean value pre } \\
\text { op and post op } \\
\text { in smokers }\end{array}$ & $\begin{array}{c}\text { Difference in } \\
\text { mean value ore } \\
\text { op and post op } \\
\text { in non smokers }\end{array}$ \\
\hline Need to blow nose & 1.41 & 1.33 \\
\hline Nasal blockage & 1.52 & 1.42 \\
\hline Sneezing & 1.38 & 1.34 \\
\hline Runny nose & 1.42 & 1.4 \\
\hline Cough & 1.1 & 0.94 \\
\hline Post nasal discharge & 1.1 & 1.24 \\
\hline Thick nasal discharge & 1.35 & 1.47 \\
\hline Ear fullness & 1.35 & 1.41 \\
\hline Dizziness & 0.76 & 0.9 \\
\hline Ear pain & 0.79 & 1.21 \\
\hline Facial pain & 1.14 & 1.30 \\
\hline Decreased taste/smell & 1.27 & 1 \\
\hline Difficulty falling asleep & 1.07 & 0.81 \\
\hline Wake up night & 0.55 & 0.7 \\
\hline Lack of a good night's sleep & 1.07 & 0.77 \\
\hline Wake up tired & 1.07 & 1.01 \\
\hline Fatigue & 1.97 & 2.03 \\
\hline Reduced productivity & 1.14 & 1.14 \\
\hline Reduced concentration & 0.96 & 0.78 \\
\hline Frustrated/irritable & 0.52 & 0.86 \\
\hline Sad & 0.76 & 0.93 \\
\hline Embarrassed & 0.83 & 0.79 \\
\hline
\end{tabular}

\begin{tabular}{|l|c|c|}
\hline Group & Preop score & Postop score \\
\hline Not tried quitting smoking & 50.50 & 27.71 \\
\hline Tried quitting smoking & 48.53 & 24.93 \\
\hline
\end{tabular}

Table 4: Comparison of total SNOT 22 scores among smokers, who had tried quitting smoking and not tried quitting smoking.

\section{Discussion}

In our study population, 61 patients were males and 39 were females. Similar gender distribution is seen in study conducted by Bezerra., et al. (Male: Female - 1.5:1) [9]. 29 patients had the habit of smoking at the time of surgery and continued to smoke at the time of answering the questionnaire post operatively at six months. 27 out of 29 smokers were males (93.10\%) and 2 out of 29 were females (6.90\%). This is in accordance with the study conducted by Briggs., et al. were 26 out of 82 were smokers (31.70\%) [10].

The average SNOT 22 score was 47.38 preoperatively among smokers which fell to 26.28 at the end of 6 months of follow up post operatively (P value $<0.0001$ ) showing FESS was statistically significant in improving quality of life among CRS patients who were smokers. Among non smokers the average SNOT 22 score was 49.99 and 26.88 pre and post operatively respectively (P value $<0.0001$ ) showing FESS was equally effective in non smokers also in improving the quality of life. Study conducted by Shivkumar., et al. showed improvement in all symptoms associated with CRS after FESS with P value $<0.001$ [11].

Nasal blockage was the commonest symptom which was reported by all the patients with average pre operative score 3.24 and 3.03 among smokers and non smokers which fell to 1.72 and 1.61 post operatively respectively. The next common symptom noted was thick nasal discharge which was seen in 96 patients with average score of 2.90 and 2.85 among smokers and non smokers, however postoperatively the average scores were 1.55 and 1.38 respectively. In the study conducted by Shivkumar, et al. nasal blockage was the commonest symptom (86\%) followed by anosmia (77\%). Following FESS symptomatic improvement was noted with all symptoms. Improvement in nasal blockage and nasal discharge was noted among 94\% and $89 \%$ patients in our study, this observation is in accordance with a study by Shivkumar., et al. which showed $89 \%$ and $87 \%$ improvement with nasal blockage and discharge respectively [11].

Table 3: Comparison of mean value difference in both groups. 
We undertook the study after noticing in patient population that smoking patients are more likely to experience symptoms associated with CRS even after undergoing FESS and thus hypothesized that FESS outcome would have been worse in smokers as compared to non smokers. This is supported by the study by Senior., et al. in which they mentioned a higher number of revision endonasal procedures noted among smokers as compared to non smokers [12]. A similar study done by Briggs., et al. showed that SNOT 16 scores were higher in smokers at the end of $6-12^{\text {th }}$ month of post FESS surgery [10]. In our study, smokers experienced slight symptomatically better improvement after FESS indicated by the change in SNOT 22 from pre op value of 49.38 to 26.28 post operatively as compared to non smokers (preop value-49.99, post op value 26.87). According to the study conducted by Briggs., et al. smoking was associated with statisticaly higher post operative SNOT score.18 These differences in findings in the present study and the study by Briggs., et al. can be attributed to the differences in sample size, average number of cigarettes and average duration of smoking [10].

On evaluation of each symptom score, the improvement was significantly more in non smoking group for thick nasal discharge, ear fullness, ear ache, dizziness, facial pain/pressure as compared to smoking group post surgery. The sleep related problems such as difficulty in falling asleep, lack of a good night sleep, waking up tired and fatigue, improved more in smoking group as compared to nonsmoking group. In a study conducted by Alt., et al. tobacco smokers found to have poor average Pittsburgh Sleep Quality Index (PQLI) score as compared to non smokers in patients with CRS [13]. The impact of cessation of smoking in reversing the disease progression has to be evaluated, however in this study there was no statistically significant difference in patients who had tried quitting smoking at any point of their life time and those who had not tried quitting smoking.

As CRS is a multi factorial condition, several other factors like allergic rhinitis, alcohol, other environmental toxins etc. can be the contributing factors. The effect of passive smoking was also not considered in the study; however non smokers who had history of passive smoking were excluded from the study.

\section{Conclusion}

Functional endoscopic sinus surgery was found to be effective in improving quality of life in patients with chronic rhinosinusitis and the SNOT 22 scoring system can be used as a tool in assessing the post operative outcome of FESS. Although smoking has not showed any significant difference in outcome after FESS as compared with non-smoking group, the short duration of follow up ( 6 months) can be a possible reason for the same. Improvement in post surgery period cannot be ruled out in the long run. Even though no studies have proven the effect of withdrawal of smoking on reversibility of mucosal injury in sinonasal cavity, it is better to quit the habit of smoking because of its proven impact on MCC.

\section{Acknowledgements}

Authors would like to acknowledge the service of Dr. Nair Tara who helped during the research, providing language help and proof reading of the article.

\section{Funding}

The research did not receive any specific grant from funding agencies in the public, commercial, or not-for-profit sectors.

\section{Conflict of Interest}

Nil.

\section{Bibliography}

1. Philpott Carl. "Rhinosinusitis; Definitions, Classification and Diagnosis". Scott-brown's Otorhinolaryngology, head and neck surgery, edited by John C Watkinson and Raymond W Clarke, $8^{\text {th }}$ Ed, Edward arnold publishers ltd, Great Britain 1 (2017): 1025-1034.

2. Kern Robert C and Liddy Whitney. "Pathogenesis of chronic rhinosinusitis". Cummings otolaryngology- head and neck surgery, edited by Flint, Paul W., et al. 6th ed, Elsevier, Canada (2015): 714-724.

3. Lal Devyani and Stankiewicz James A. "Primary sinus surgery". Cummings otolaryngology- head and neck surgery, edited by Flint, Paul W., et al. 6th ed, Elsevier, Canada (2015): 752-782.

4. Leopold Philip L., et al. "Smoking is associated with shortened airway cilia”. Plos One 4.12 (2009): e8157.

5. Baby Manu Kurian., et al. "Effect of cigarette smoking on nasal mucociliary clearance: A comparative analysis using saccharin test". Lung India: Official organ of Indian Chest Society 31.1 (2014): 39-42.

6. Damm Michael., et al. "Impact of functional endoscopic sinussurgery on symptoms and quality of life in chronic rhinosinusitis". The Laryngoscope 112,2 (2002): 310-315. 
7. Vennik Jane., et al. "Chronic rhinosinusitis: a qualitative study of patient views and experiences of current management in primary and secondary care". BMJ Open 9.4 (2019): e022644.

8. Schaffer SD., et al. "Endoscopic paranasal sinus surgery: indications and considerations". The Laryngoscope 99.1 (1989): $1-5$.

9. Bezerra Thiagro Freire Pinto., et al. "Assessment of quality of life after endoscopic sinus surgery for chronic rhinosinusitis". Brazilian Journal of Otorhinolaryngology 78.2 (2012): 96-102.

10. Briggs Russel D., et al. "Smoking in Chronic Rhinosinusitis: A Predictor of Poor Long-Term Outcome after Endoscopic Sinus Surgery". The Laryngoscope 114.1 (2004): 126-128.

11. Shivkumar T and AP Sambandan. "Retrospective Analysis of the Effectiveness of Functional Endoscopic Sinus Surgery in the Treatment of Adult Chronic Rhinisinusitis Refractory to Medical Treatment". Indian Journal of Otolaryngology and Head and Neck Surgery: Official Publication of Otolaryngologists of India 63.4 (2011): 321-324.

12. Senior B A., et al. "Long-term results of functional endoscopic sinus surgery". The Laryngoscope 108.2 (1998): 151-157.

13. Alt Jeremiah., et al. "Sleep quality and disease severity in patients with chronic rhinosinusitis". The Laryngoscope 123.10 (2013): 2364-2370.

Volume 3 Issue 12 December 2021

(C) All rights are reserved by Ashok Vivekanand and Lyra Joy. 